
\title{
Incidência de úlcera por pressão em pacientes
neurocirúrgicos de hospital universitário*
}

\author{
The incidence of pressure ulcer in neurosurgical patients from a university hospital \\ Incidencia de úlcera por decúbito en pacientes neuroquirúrgicos de un hospital universitario
}

Solange Diccini ${ }^{1}$, Camila Camaduro ${ }^{2}$, Luciana Inaba Senyer Iida $^{3}$

\begin{abstract}
RESUMO
Objetivo: Conhecer a incidência de úlcera por pressão no pré e pós-operatório de pacientes neurocirúrgicos e descrever as medidas preventivas implementadas pela equipe de enfermagem durante nesses períodos. Métodos: Estudo de Coorte prospectivo realizado na unidade de neurocirurgia do Hospital São Paulo. Foram incluídos pacientes submetidos a cirurgias eletivas do sistema nervoso central e que não apresentaram úlcera por pressão na internação. Resultados: Foram avaliados 60 pacientes sendo que 34 (56,6\%) do sexo feminino. A mediana da idade foi de 49 anos, com variação de 19 a 78 anos. Oito (13,4\%) pacientes desenvolveram nove úlceras por pressão. Dos pacientes de risco para úlcera por pressão, três (25\%) no pré-operatório, 33 (66\%) no pós-operatório imediato e 13 (86,6\%) no pósoperatório mediato tinham medidas de prevenção para úlcera por pressão. Conclusão: A incidência de úlcera por pressão foi de 13,3\%. Há necessidade de implantação de programas de prevenção, com a finalidade de diminuir a úlcera por pressão em pacientes neurocirúrgicos.

Descritores: Úlcera de pressão; Enfermagem; Incidência; Procedimentos neurocirúrgicos
\end{abstract}

\begin{abstract}
Objective: To identify the incidence of pressure ulcer in the preoperative and postoperative period in neurosurgical patients and to describe preventive measures adopted by nursing staff. Methods: A prospective study was conducted in the neurosurgical unit of the Sao Paulo hospital. The sample consisted of inpatients who were admitted for elective surgeries of the central nervous system and who did not have pressure ulcer. Results: Sixty patients participated in the study. They have a mean age of 49 years, ranging from 19 to 78 years. The majority of patients (56.6\%) were female. Only 8 patients (13.4\%) developed nine pressure ulcers. Among the patients identified as having risk for pressure ulcers, preventive measures were implement to 3 of them (25\%) during the preoperative period, to 33 of them (66\%) during the immediate postoperative period, and to $23(86.6 \%)$ during the mediate postoperative period. Conclusion: The incidence of pressure ulcer in the study sample was $13.3 \%$. There is a need to implement a preventive program to decrease the incidence of pressure ulcers in neurosurgical patients.
\end{abstract}

Keywords: Pressure ulcer; Nursing; Incidence; Neurosurgical procedures

\section{RESUMEN}

Objetivo: Conocer la incidencia de úlcera por decúbito en el pre y post operatorio de pacientes neuroquirúrgicos y describir las medidas preventivas implementadas por el equipo de enfermería durante esos períodos. Métodos: Estudio de Cohorte prospectivo realizado en la unidad de neurocirugía del Hospital São Paulo. Fueron incluidos pacientes sometidos a cirugías electivas del sistema nervioso central y que no presentaron úlcera por decúbito en el internamiento. Resultados: Se evaluaron 60 pacientes de los cuales 34 (56,6\%) eran del sexo femenino. El promedio de edad fue de 49 años, con variación de 19 a 78 años. Ocho (13,4\%) pacientes desarrollaron nueve úlceras por decúbito. De los pacientes de riesgo para úlcera por decúbito, tres (25\%) en el pre-operatorio, 33 (66\%) en el post-operatorio inmediato y 13 $(86,6 \%)$ en el post-operatorio mediato tenían medidas de prevención para úlcera por decúbito. Conclusión: La incidencia de úlcera por decúbito fue del 13,3\%. Hay necesidad de implantación de programas de prevención, con la finalidad de disminuir la úlcera por decúbito en pacientes neuroquirúrgicos.

Descriptores: Úlcera por presión; Enfermería; Incidencia; Procedimientos neuroquirúrgicos

\footnotetext{
* Estudo realizado nas unidades de internação e de terapia intensiva da neurocirurgia do Hospital São Paulo da Universidade Federal de São Paulo - UNIFESP - São Paulo (SP), Brasil.

${ }^{1}$ Professora Adjunto do Departamento de Enfermagem da Universidade Federal de São Paulo - UNIFESP, São Paulo (SP), Brasil.

${ }^{2}$ Especialista em Enfermagem Clínica e Cirúrgica e em Enfermagem em Epidemiologia Clínica pela Universidade Federal de São Paulo - UNIFESP, São Paulo (SP), Brasil.

${ }^{3}$ Enfermeira da UTI adulto do Hospital Universitário da Universidade de São Paulo, Pós-graduanda do Programa de Pós-graduação do Departamento de Enfermagem da Universidade Federal de São Paulo - UNIFESP, São Paulo (SP), Brasil.
} 


\section{INTRODUÇÃO}

Úlcera por pressão (UP) é definida como uma lesão localizada na pele e/ ou no tecido ou estrutura subjacente, geralmente sobre uma proeminência óssea, resultante de pressão isolada ou de pressão combinada com fricção e/ou cisalhamento ${ }^{(1)}$.

Em estudo multicêntrico realizado com 843 pacientes, a incidência de UP foi de 8,5\% em hospitais universitários, de $7,4 \%$ em centros médicos e de $23,9 \%$ em casas de repouso $^{(2)}$. Estudos nacionais verificaram incidência de UP entre $17,7 \%$ e $39,8 \%{ }^{(3-4)}$.

O tratamento das UP, sobretudo em estágios avançados, pode ser demorado e de alto custo, sendo que em alguns casos, a intervenção cirúrgica torna-se necessária. No Brasil não existem dados precisos do custo das UP para o sistema de saúde. Estudos internacionais demonstram que cada lesão pode custar de 2.000 a 30.000 mil dólares, dependendo do estágio, podendo chegar a 1,3 bilhões de dólares anuais( ${ }^{(5)}$.

Fatores intrínsecos e extrínsecos podem contribuir para o aparecimento das UP. Os fatores intrínsecos são determinados pelas condições do paciente, tais como: estado nutricional alterado, incontinência urinária e fecal, hipertermia, tabagismo, idade avançada, insuficiência arterial ou venosa e diabetes mellitus. A pressão exercida sobre um tecido, bem como a sua duração e intensidade são descritos com importantes fatores extrínsecos ${ }^{(3,6)}$.

Os pacientes neurológicos podem apresentar seqüelas motoras como paresia ou plegia em um membro ou num hemicorpo, que prejudicam a mobilidade, causando muitas vezes a restrição do paciente no leito. Alterações de sensibilidade dolorosa e tátil podem prejudicar a avaliação pelo paciente, de regiões que estão sob pressão. A UP em pacientes neurológicos pode ocorrer devido a fatores de risco como: imobilidade, incontinência urinária e fecal, alteração do nível de consciência, fraturas e alteração sensitiva e cognitiva ${ }^{(7)}$. Além disso, alteração do sono e repouso, perfusão tissular alterada e déficit nutricional são características do paciente neurológico consideradas de risco para desenvolver $\mathrm{UP}^{(8)}$.

As UP são a causa mais comum de complicação que acomete os pacientes com lesão medular, a incidência varia entre $7,5 \%$ e $31,7 \%$ em unidades de reabilitação ${ }^{(9)}$. Em unidades de internação a incidência de UP em pacientes com lesão medular variou de $42,5 \%$ e $63 \%{ }^{(10-11)}$. Outro estudo verificou que cerca de $60 \%$ dos pacientes tetraplégicos hospitalizados desenvolveram lesão de pele ${ }^{(9)}$.

Em unidade neurocirúrgica 16,6\% dos pacientes desenvolveram UP durante a internação hospitalar ${ }^{(12)}$. Outro estudo verificou que a incidência de UP em pacientes neurocirúrgicos foi de $9,5 \%{ }^{(13)}$. Quando foram avaliados pacientes internados numa unidade de terapia intensiva neurológica a incidência de UP foi de $12,4 \%{ }^{(7)}$.
Cerca de $50 \%$ a $80 \%$ dos indivíduos com lesão medular desenvolveram pelo menos uma lesão de pele no decorrer da vida ${ }^{(14)}$, e foi mostrado que este risco aumentava em média $4,2 \%$ a cada ano ${ }^{(9)}$.

Nestes anos de atuação com pacientes internados na unidade de neurocirurgia, verificamos que os pacientes, na admissão, podem apresentar alterações no exame neurológico, como alterações na força motora, sensibilidade e dos nervos cranianos, que colocam o paciente em risco para UP. Porém, observamos também que no pós-operatório, devido ao tratamento cirúrgico, pacientes sem risco prévio de UP no pré-operatório, apresentavam risco para UP no pós-operatório. Devido a importância do assunto e da escassez de estudos publicados sobre UP em pacientes neurocirúrgicos foi realizado este estudo cujo objetivo foi conhecer a incidência de UP no pré e pós-operatório de pacientes neurocirúrgicos e descrever as medidas preventivas implementadas pela equipe de enfermagem nesses períodos.

\section{MÉTODOS}

Estudo de coorte prospectivo realizado nas unidades de internação e de terapia intensiva da neurocirurgia do Hospital São Paulo da Universidade Federal de São Paulo (UNIFESP), no período de novembro de 2002 a janeiro de 2004. Os critérios de inclusão foram: pacientes com idade igual ou maior a 18 anos, pacientes conscientes no pré-operatório e pacientes internados na unidade de internação com doenças neurocirúrgicas eletivas. Foram excluídos os pacientes que apresentavam UP no momento da admissão na unidade de internação. $O$ projeto de pesquisa foi aprovado pelo Comitê de Ética em Pesquisa da UNIFESP, sob o número 1054/02. Os pacientes foram informados quanto aos objetivos do estudo e foram incluídos após assinatura do Termo de Consentimento Livre e Esclarecido.

Os pacientes foram avaliados na admissão no préoperatório de cirurgias intracranianas ou de coluna vertebral, e durante o pós-operatório nas unidades de terapia intensiva e de internação da neurocirurgia. A coleta de dados foi realizada diariamente pelas pesquisadoras nos pacientes das unidades de internação e de terapia intensiva da neurocirurgia. Foram preenchidos os seguintes instrumentos: identificação do paciente, escala de Braden e classificação dos estágios da UP. No instrumento de identificação do paciente foram coletados dados sobre: sexo, idade, diagnóstico médico e tempo de internação no pré e no pós-operatório.

A escala de Braden, em sua versão adaptada e validada para o Brasil ${ }^{(15)}$, foi o instrumento utilizado para avaliação de risco para o desenvolvimento de UP e foi aplicada diariamente durante o período de pré e pós-operatório. 
Durante o período da coleta de dados, a escala de Braden não era utilizada pelas enfermeiras nas unidades de internação e de terapia intensiva. Devido a este motivo foram as pesquisadoras que aplicaram diariamente a escala. Atualmente a escala de Braden já é aplicada pelas enfermeiras onde o estudo foi realizado.

Esta escala possui seis subescalas que refletem os determinantes críticos de pressão (mobilidade, atividade e percepção sensorial) e fatores que influenciam na tolerância da pele à pressão (umidade da pele, estado nutricional, fricção e cisalhamento). Cada subescala é graduada de 1 a 3 ou 4 pontos, num total máximo de 23 pontos. Uma baixa pontuação na escala de Braden indica um alto risco de o paciente desenvolver UP. Para pacientes adultos hospitalizados, uma contagem menor ou igual a 16 pontos foi considerada de risco para o desenvolvimento de UP. Uma pontuação de 16 foi considerada de risco mínimo; de 13 a 14 de risco moderado e de 12 ou menos pontos, de risco elevado ${ }^{(15)}$. Após a avaliação do risco para UP, foram anotadas as medidas de prevenção prescritas pelas enfermeiras e checadas pela equipe de enfermagem das unidades de internação e de terapia intensiva.

Em relação às intervenções de enfermagem na prevenção da $\mathrm{UP}^{(1)}$ nos pacientes de risco, as pesquisadoras anotaram, a partir da prescrição de enfermagem, as seguintes intervenções: utilização de colchão piramidal, hidratação da pele com creme hidratante e mudança de decúbito de duas em duas horas.

$\mathrm{Na}$ coleta de dados foi incluída a presença ou ausência de UP, localização e estadiamento da UP, bem como, o local de internação que o paciente desenvolveu a UP. A UP foi classificada, segundo a proposta do National Pressure Ulcer Advisory Panel ${ }^{(1)}$, em 4 estágios (I a IV). A coleta de dados foi encerrada quando da alta hospitalar ou óbito dos pacientes.

Para análise estatística foi utilizado o programa SPSS for Windows na versão 10.0. As variáveis quantitativas foram analisadas por meio da observação dos valores mínimos e máximos e do cálculo da mediana. Para as variáveis qualitativas, calcularam-se as freqüências absolutas e relativas. Para análise da hipótese de igualdades de médias entre os dois grupos, foi utilizado o teste de t de Student; quando a suposição de normalidade dos dados foi rejeitada, utilizouse o teste não-paramétrico de Mann-Whitney. Para testar a homogeneidade entre as proporções dos dois grupos, foi utilizado o teste qui-quadrado ou o teste exato de Fisher. O nível de significância utilizado para os testes foi de 5\%.

\section{RESULTADOS}

Neste estudo foram incluídos 60 pacientes sendo que $34(56,6 \%)$ eram do sexo feminino. A mediana da idade dos pacientes foi de 49 anos, variando de 19 a 78 anos.
Os diagnósticos médicos encontrados foram: 30 pacientes com tumores intracranianos $(50 \%), 8$ pacientes com aneurisma cerebral $(13,5 \%), 5$ pacientes com malformação arteriovenosa (8,4\%), 4 pacientes com hérnia de disco (6,6\%), 4 pacientes com tumores medulares (6,6\%), 3 pacientes com trauma raquimedular $(5,0 \%), 2$ pacientes com abcesso cerebral $(3,3 \%), 2$ com hidrocefalia $(3,3 \%)$ e 2 pacientes com neurocisticercose $(3,3 \%)$.

$\mathrm{Na}$ admissão dos pacientes na unidade de internação da neurocirurgia, após a avaliação do risco pela Escala de Braden, $12(20,0 \%)$ pacientes tinham risco para desenvolver UP no pré-operatório. A pontuação mediana pela Escala de Braden destes pacientes foi de 14,5 pontos, variando de 10 a 16 pontos. Em três pacientes (25\%) foram adotadas medidas de prevenção para UP, sendo que um paciente foi submetido à mudança de decúbito de duas em duas horas e colchão piramidal e dois pacientes foram submetidos à mudança de decúbito de duas em duas horas e hidratação da pele com creme hidratante. Dos 12 pacientes com risco no pré-operatório, nenhum paciente evoluiu com UP.

Na Tabela 1 são apresentados os tempos de préoperatório, cirúrgico e de pós-operatório dos pacientes com risco e sem risco para UP pela Escala de Braden. Pelo teste de Mann-Whitney não houve diferença estatisticamente significante entre os tempos de pré-operatório e cirúrgico dos pacientes com risco e sem risco para UP. Pelo teste de Mann-Whitney, houve relação estatisticamente significante entre o tempo de pós-operatório dos pacientes com risco e sem risco para UP, sendo que os pacientes com risco para UP tiveram maior tempo de internação no pósoperatório. Em relação ao número de pacientes com risco e sem risco para UP no pré-operatório e no pós-operatório, pelo teste de qui-quadrado, não houve diferença estatisticamente significante entre os grupos $(p=0,26)$.

Após o término da cirurgia, 39 pacientes foram encaminhados para a unidade de terapia intensiva da neurocirurgia $(65 \%)$ e 21 para a unidade de internação neurocirurgia (35\%), para o pós-operatório imediato. Realizada a avaliação de risco para UP pela Escala de Braden, 50 pacientes apresentaram risco para UP (83,3\%), com uma pontuação mediana pela Escala de Braden de 12 pontos, variando de 8 a 16 pontos. Dos pacientes de risco 33 receberam medidas de prevenção (66\%), sendo que 14 receberam hidratação da pele com creme hidratante e colchão piramidal (28\%), 11 receberam mudança de decúbito de duas em duas horas e hidratação da pele com creme hidratante $(22 \%), 5$ receberam mudança de decúbito de duas em duas horas e hidratação da pele com creme hidratante $(10 \%)$, dois receberam mudança de decúbito de duas em duas horas e colchão piramidal (4\%) e apenas um paciente recebeu as três medidas de prevenção associadas (2\%). Durante o pós-operatório imediato, nenhum paciente apresentou UP. 
Tabela 1 - Pacientes segundo tempo de pré-operatório, com risco e sem risco para úlcera de pressão pela Escala de Braden, na Unidade de Neurocirurgia do Hospital São Paulo/UNIFESP - 11/2002 a 01/2004

\begin{tabular}{lcccc}
\hline Tempo & Total & Com risco UP & Sem risco UP & Valor de $\mathbf{p}$ \\
\hline Pré-opera tório (dias) & $\mathrm{n}=60$ & $\mathrm{n}=12$ & $\mathrm{n}=48$ \\
Mediana (variação) & $7,0(1-27)$ & $8,5(1-27)$ & $5,5(1-23)$ & 0,26 \\
Cirúrgico (minutos) & $\mathrm{n}=60$ & $\mathrm{n}=8$ & $\mathrm{n}=52$ & \\
Mediana (variação) & & $435(270-600)$ & $360(70-840)$ & 0,05 \\
Pós-operatório (dias) & $\mathrm{n}=59$ & $\mathrm{n}=15$ & $\mathrm{n}=44$ & \\
Mediana (variação) & $8(1-68)$ & $16(2-68)$ & $6(1-27)$ & $0,0001^{*}$ \\
\hline $\begin{array}{l}\text { UP= úlcera de pressão } \\
\text { * Teste de Mann-Whitney, estatisticamente significante. }\end{array}$
\end{tabular}

No pós-operatório mediato, um paciente evoluiu para óbito, após alta da UTI, devido à embolia pulmonar (1,7\%). Dos 59 pacientes que permaneceram no estudo, 15 foram considerados de risco para desenvolver UP $(25,4 \%)$, apresentando pela Escala de Braden a pontuação mediana de 10,5 pontos, variando de 8 a 16 pontos. As medidas de prevenção foram aplicadas em 13 pacientes de risco $(86,6 \%)$, sendo que 5 pacientes receberam colchão piramidal e hidratação da pele com creme hidratante $(38,5 \%), 4$ receberam mudança de decúbito de duas em duas horas e hidratação da pele com creme hidratante $(30,8 \%)$, dois receberam somente hidratação da pele com creme hidratante $(15,4 \%)$, um paciente recebeu mudança de decúbito de duas em duas horas e colchão piramidal $(7,7 \%)$ e um paciente recebeu as três medidas associadas $(7,7 \%)$.

Dos 59 pacientes avaliados, oito pacientes evoluíram com UP, sendo a incidência de UP em pacientes neurocirúrgicos no pós-operatório de 13,6\%. Dos pacientes com UP, seis pacientes desenvolveram a UP na UTI de neurocirurgia e dois na unidade de internação da neurocirurgia. Dos 15 pacientes que tinham risco para UP no pós-operatório mediato, oito apresentaram UP $(53,3 \%)$. Destes pacientes, cinco eram do sexo feminino e três do sexo masculino.

Dos oito pacientes que apresentaram UP no pósoperatório mediato, sete pacientes apresentaram a UP em uma região do corpo, enquanto um paciente apresentou duas UP em dois locais diferentes do corpo, maléolo e trocanter, num total de nove UP. Três dos pacientes desenvolveram UP na região do maléolo; dois pacientes no calcâneo, um no trocanter, um na região sacral, um na crista ilíaca e um na região occipital.

$\mathrm{Na}$ Figura 1 verifica-se a distribuição das UP em relação a localização e ao estágio. Houve predomínio de UP na região do maléolo. Do total das UP, cincoforam UP Estágio I, duas foram Estágio II, uma foi Estágio III e uma foi Estágio IV.

Segundo o dia de instalação da UP no pós-operatório mediato, observou-se que entre o $1^{\circ}$ e $4^{\circ}$ dia pós-ocorreu uma UP; entre o $4^{\circ}$ e $8^{\circ}$ dias ocorreram quatro UP, entre o $8^{\circ}$ e o $12^{\circ}$ dias ocorreram duas UP e acima do $12^{\circ}$ dia de pós-operatório foram observadas duas UP. Houve um predomínio de UP entre o $4^{\circ}$ e o $8^{\circ}$ dia de pósoperatório.

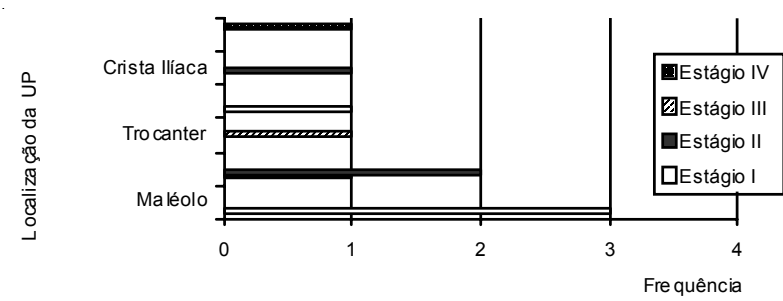

Figura 1 - Distribuição das UP, segundo localização e estágio em pacientes de Unidade de Neurocirurgia do Hospital São Paulo/UNIFESP - 11/2002 a 01/2004

\section{DISCUSSÃO}

A mudança de decúbito, hidratação da pele com creme hidratante e o colchão piramidal são algumas medidas preventivas para UP de fácil operacionalização. Porém, a utilização do colchão piramidal depende da sua compra pela instituição, enquanto a mudança de decúbito e a hidratação da pele dependem exclusivamente da prescrição realizada pela enfermeira e das intervenções realizadas pela equipe de enfermagem. Neste estudo, no pós-operatório, dos 15 pacientes que apresentavam risco para UP, dois pacientes não tinham prescrição de enfermagem das medidas preventivas e apenas um deles recebeu as três medidas associadas.

As regiões mais freqüentes para o desenvolvimento de UP em hospitais são: calcâneos, sacroccígea e glúteo. A incidência de UP na região sacra pode variar de 30,5\% a 33,6\%, nos glúteos de $11,8 \%$ a $23,9 \%$ e nos calcâneos $24,6 \%$ a $42,4 \%{ }^{(4,9)}$. Neste estudo as regiões predominantes de UP foram o maléolo e calcâneo.

Em pacientes com lesão traumática a ocorrência de UP foi de $63 \%$, sendo que $36 \%$ eram lesões na região sacra e $22 \%$ nos calcâneos ${ }^{(11)}$. As lesões eram predominantemente de estágio II (39,0\%) seguidas pelas lesões de estágio I $(17,0 \%)^{(16)}$. Neste estudo, $55,5 \%$ dos pacientes com UP tiveram estágio I e 22,3\% estágio II, o que facilitou o tratamento e a reversão da lesão, bem como, a diminuição do custo e do tempo de internação, 
quando comparado a UP de estágios III e IV.

Neste estudo o tempo de internação no pósoperatório dos pacientes com risco para o desenvolvimento de UP foi de 16 dias e para os que não possuíam risco foi de seis dias. Estudo mostrou que a média de tempo de hospitalização para pacientes cirúrgicos era de sete dias, enquanto os pacientes cirúrgicos que desenvolveram UP foi de 16,5 dias. ${ }^{(17)}$ Em pacientes com lesão medular uma UP acrescenta, em média, 42 dias na hospitalização ${ }^{(7)}$.

O desenvolvimento e o aparecimento das UP neste estudo ocorreram em $44,5 \%$ no período compreendido entre o $4^{\circ}$ e o $8^{\circ}$ dia de pós-operatório, dado similar foi encontrado em outro estudo que mostrou que o tempo médio de aparecimento das lesões foi de 4,7 dias ${ }^{(3)}$.

A incidência de UP encontrada neste estudo foi de 13,3\%, o que não diferiu dos poucos estudos encontrados na literatura que avaliaram pacientes neurocirúrgicos ${ }^{(7,12-13)}$.

O conhecimento da incidência de UP na unidade de neurocirurgia foi o primeiro passo para determinar a dimensão do problema e estimular a equipe de enfermagem para que medidas sejam tomadas com a finalidade de diminuir esta taxa. A implantação da avaliação de risco pela utilização sistemática da Escala de Braden e investimento em capacitação da equipe de enfermagem são medidas necessárias para a melhoria da qualidade da assistência de enfermagem prestada.

Estudo realizado com pacientes mostrou que a incidência de UP foi reduzida de $14 \%$ para $6 \%$, depois

\section{REFERÊNCIAS}

1. National Pressure Ulcer Advisory Panel [Internet]. Updated pressure ulcer staging 2007. [cited 2008 Apr 10]. Available from: http:/ /www.npuap.org/resources.htm.

2. Meraviglia M, Becker H, Grobe SJ, King M. Maintenance of skin integrity as a clinical indicator of nursing care. Adv Skin Wound Care. 2002;15(1):24-9.

3. Anselmi ML, Peduzzi M, França Júnior I. Estudo da incidência de úlceras de pressão segundo cuidado de enfermagem. Rev Formação. 2003;3(7):57-72.

4. Rogenski NMB, Santos VLCG. Estudo sobre a incidência de úlceras de pressão em um hospital universitário. Rev Latinoam Enferm. 2005;13(4):474-80.

5. Lewis M, Pearson A, Ward C. Pressure ulcer prevention and treatment: transforming research findings into consensus based clinical guidelines. Int J Nurs Pract. 2003;9(2):92-102.

6. Souza DMST, Santos VLCG. Fatores de risco para o desenvolvimento de úlceras por pressão em idosos institucionalizados. Rev Latinoam Enferm. 2007;15(5):958-64.

7. Fife C, Otto G, Capsuto EG, Brandt K, Lyssy K, Murphy K, Short C. Incidence of pressure ulcers in a neurologic intensive care unit. Crit Care Med. 2001;29(2):283-90.

8. Costa IG, Caliri MHL. Incidência de úlcera de pressão em centro de terapia intensiva de um hospital universitário e fatores de risco relacionados. Rev Paul Enferm. 2005;23(3/4): 202-7.

9. New PW, Rawicki HB, Bailey MJ. Nontraumatic spinal cord injury rehabilitation: pressure ulcer patterns, prediction, and impact. Arch Phys Med Rehabil. 2004;85(1):87-93. da implementação de um programa de educação para a equipe multiprofissional ${ }^{(2)}$.

Uma das limitações do estudo foi a exclusão de pacientes inconscientes, complicação esta encontrada tanto em pacientes no pré-operatório, bem como no pósoperatório de neurocirurgia. Pacientes com alterações no nível de consciência e inconscientes são de risco para UP.

\section{CONCLUSÕES}

A incidência de ulcera de pressão em pacientes neurocirúrgicos foi de $13,3 \%$, sendo que o desenvolvimento das úlceras de pressão ocorreu no período de pós-operatório mediato e a maioria das lesões desenvolveu-se na unidade de terapia intensiva neurocirúrgica. Em relação às medidas de prevenção de UP, nos pacientes de risco, no pré-operatório nenhum paciente recebeu as três medidas. Tanto no pós-operatório imediato como no pós-operatório mediato, apenas um paciente recebeu as três medidas de prevenção para UP.

Apesar da escassez de recursos em relação ao colchão piramidal, que a equipe de enfermagem tinha para realizar a prevenção da UP, a utilização de mudança sistemática de decúbito e hidratação da pele com creme hidratante, foram medidas eficazes para que esta incidência permanecesse dentro do limite apresentado pela literatura. Porém, há necessidade de implantação de programas de prevenção, com a finalidade de diminuir a taxa de UP em pacientes neurocirúrgicos.
10. Nogueira PC, Caliri MHL, Haas VJ. Profile of patients with spinal cord injuries and occurrence of pressure ulcer at a university hospital. Rev Latinoam Enferm. 2006;14(3):372-7.

11. Carcinoni M, Caliri MHL, Nascimento MS. Ocorrência de úlcera de pressão em indivíduos com lesão traumática da medula espinhal. REME Rev Min Enferm. 2005;9(1):29-34.

12. Michelone APC, Cardoso LAA, Motta RP, Borella AC, Mello AP. Incidência de úlceras por pressão no Hospital de Clínicas I da Faculdade de Medicina de Marília - SP. Rev Esc Enferm USP. 1999;33(Nº Esp):207-10.

13. Chang CH, Chen HL, Chen HC. Program for lowering the incidence of pressure sores in neurosurgical patients]. Hu Li Za Zhi. 2007;54(6):53-60. Chinese.

14. Schultz A, Bien M, Dumond K, Brown K, Myers A. Etiology and incidence of pressure ulcers in surgical patients. AORN J. 1999;70(3):434,437-40,443-9.

15. Paranhos WY, Santos VLCG. Avaliação de risco para úlceras de pressão por meio da Escala de Braden, na língua portuguesa. Rev Esc Enferm USP. 1999;33( No Esp):191-206

16. Amlung SR, Miller WL, Bosley LM. The 1999 National Pressure Ulcer Prevalence Survey: a benchmarking approach. Adv Skin Wound Care. 2001;14(6):297-301.

17. Bergstrom N, Braden B, Kemp M, Champagne M, Ruby E. Multi-site study of incidence of pressure ulcers and the relationship between risk level, demographic characteristics, diagnoses, and prescription of preventive interventions. J Am Geriatr Soc. 1996;44(1):22-30. 